\title{
Lineamientos para un método de enseñanza de quechua para bilingües receptivos en quechua ${ }^{1}$
}

\section{Guidelines for a Quechua Teaching Method for Receptive Bilinguals in Quechua}

\author{
Norma Meneses Tutaya \\ Universidad Nacional Mayor de San Marcos, \\ Lima, Perú \\ https://orcid.org/oooo-00o2-0622-5783 \\ nmenesest@unmsm.edu.pe
}

\author{
María Mercedes Gonzales Rodríguez \\ Universidad Nacional Mayor de San Marcos, \\ Lima, Perú \\ https://orcid.org/oooo-00o2-7078-3464 \\ mgonzalesr@unmsm.edu.pe
}

\author{
Alicia Alonzo Sutta \\ Universidad Nacional Mayor de San Marcos, \\ Lima, Perú \\ https://orcid.org/oooo-00o2-9063-508X \\ aalonzos@unmsm.edu.pe \\ Jaime Edilberto Huanca Quispe \\ Universidad Nacional Mayor de San Marcos, \\ Lima, Perú \\ https://orcid.org/oooo-00o2-0589-4942 \\ jhuancaq@unmsm.edu.pe
}

\section{Resumen}

En el presente artículo se brinda los resultados más importantes de una investigación llevada a cabo en 2017 en la región de Ayacucho que tuvo el objetivo de determinar los lineamientos teóricos y metodológicos necesarios para la elaboración de un método de enseñanza del quechua para los bilingües receptivos en quechua de esta región.

Este objetivo nos condujo a realizar un estado de la cuestión sobre los estudios de lingüística quechua, aplicación lingüística, lingüística aplicada y de estudios socioculturales sobre la cultura y la sociedad andina de esta región que sirvan de base para los mencionados lineamientos.

Adicionalmente, esta revisión nos permitió evidenciar la necesidad de nuevos estudios descriptivos de la lengua quechua, así como los aspectos a considerar en la planificación lingüística del corpus de esta lengua.

Palabras claves: enseñanza del quechua, bilingüismo receptivo, enseñanza de 1ra lengua, revitalización lingüística

1 El presente artículo corresponde a los principales resultados y conclusiones del proyecto de investigación E17030141, denominado Fundamentos de lingüística quechua, aplicación lingüistica $y$ de lingüística aplicada para la creación de un método de enseñanza de quechua para bilingües receptivos en quechua del año 2017. 
Lineamientos para un método de enseñanza de quechua para bilingües receptivos... Norma Meneses, Alicia Alonzo, María Gonzales y Jaime Huanca

\begin{abstract}
This article provides the most important results of an investigation carried out in 2017 in the Ayacucho region that had the objective of determining the theoretical and methodological guidelines necessary for a teaching method of Quechua for receptive bilinguals in Quechua from this region.

This objective led us to carry out a state of the question on the studies of Quechua linguistics, linguistic application, applied linguistics and sociocultural studies on Andean communities that serve as the basis for such guidelines.

Additionally, this review allowed us to show the need for new descriptive studies of the Quechua language and aspects of language planning of this language.
\end{abstract}

Keywords: Quechua teaching, receptive bilingualism, 1st language teaching, linguistic revitalization

Recibido: 25-06-2020

Aprobado: 27-02-2021

\title{
1. Introducción
}

La situación de las lenguas y las culturas de los pueblos andinos ha sido muy conflictiva desde el inicio de la conquista española hasta nuestros días. La evidencia de ello es la dominación y marginación política que sufrió como pueblo conquistado. Situación que no solo no cambió, sino empeoró con la República. Evidencia de ello fue que los colegios para nobles indígenas, donde aprendían a leer y escribir en latín, español y quechua los hijos de los curacas, fueron clausurados en 1781 con motivo de la revolución de Túpac Amaru II, y no fueron reabiertos después de la independencia en 1821. La educación pública solo se ofreció en castellano hasta 1990, año en que se inaugura la educación intercultural bilingüe en contadas zonas rurales de la sierra como un servicio del Estado a través del Ministerio de Educación. Todo el sistema estatal de servicios públicos se ofrece solo en lengua castellana hasta hoy. El Estado daba así la espalda a la inmensa mayoría de peruanos que hablaban el quechua y otras lenguas originarias.

Por ello, el objetivo central de las familias andinas fue aprender como fuere el castellano, la lengua del poder y de la ciudadanía. Dos fueron las soluciones que intentaron con mediano éxito: apropiarse del castellano y abandonar el quechua (sierra norte y central) o apropiarse del castellano sin perder el quechua, convirtiéndose en bilingües (sierra sur). Sin embargo, el abandono oficial de las lenguas originarias - que en el fondo es una política lingüística de dejar extinguirse a las lenguas originarias - está causando que incluso en zonas usualmente bilingües las nuevas generaciones no tiendan a conservar el carácter de bilingües activos

144 Lengua \& Sociedad. Revista de Lingüistica Teórica y Aplicada 
Lineamientos para un método de enseñanza de quechua para bilingües receptivos...

Norma Meneses, Alicia Alonzo, María Gonzales y Jaime Huanca

y lo que se observa, cada vez más, es el bilingüismo receptivo. Es decir, personas que entienden bien, pero hablan muy poco y con poca fluidez. Si esto ocurre con esta generación de niños y adolescentes, en el futuro, como padres, ellos no podrán transmitir la lengua quechua a sus hijos y entonces esta terminará siendo desplazada por el castellano. Se produce así un desplazamiento generacional de la lengua patrimonial.

El método de enseñanza quechua que se quiere elaborar va dirigido a este sector de bilingües receptivos a fin de dotarlos de las habilidades y destrezas productivas (hablar y escribir) en su L1, la lengua quechua. Indirectamente se busca generar una fuerza de reacción en defensa de la lengua y la cultura andina con su práctica oral y escrita en todo ámbito tradicional y moderno. La población que se tomó de referencia fueron los alumnos de los institutos superiores de la ciudad de Huanta en la región de Ayacucho.

El mencionado estudio tuvo como objetivo central establecer los fundamentos de lingüística quechua, aplicación lingüística, lingüística aplicada y estudios socioculturales que sirvan de base para determinar los lineamientos teóricos y metodológicos del método de enseñanza del quechua que se desea construir para los bilingües receptivos.

Con este objetivo, fue necesario evaluar el estado de los estudios de lingüística quechua, con especial atención del quechua ayacuchano y cusqueño (Cerrón Palomino, 1987; Solís y Chacón 1989; Soto, 1976; Parker, 1965; Calvo 1993; Cusihuamán, 1976) donde se pudo ver que existen buenas descripciones lingüísticas de esta lengua; sin embargo, resultan insuficientes en el área de la lingüística teórico-descriptiva oracional y textual. Aún más grande, es el vacío en la gramática normativa debido a que la planificación lingüística del corpus del quechua está incompleta. Este estudio nos ha servido para establecer los contenidos de gramática descriptiva y de gramática normativa del futuro método. Indirectamente, también ha sido útil para realizar un levantamiento de necesidades de futuras investigaciones en esta disciplina.

Con respecto a los estudios de aplicación lingüística, fue necesario revisar algunos estudios sobre temas como actitudes lingüísticas, etnografía de la comunicación, políticas lingüísticas familiares, tipos de bilingüismo individual, adquisición incompleta de L1 y atrición lingüística, (Davies, 2007; Seton y Schmid, 2016; Suárez, 2002; Szupica-Pyrzanowska, 2016; Sicra, 2006; Heijas, 2014; Von Gleich, 2014). El objetivo fue contar con un marco conceptual para la caracterización de los estudiantes de institutos superiores a quienes, en principio, irá dirigido el método. 
Lineamientos para un método de enseñanza de quechua para bilingües receptivos... Norma Meneses, Alicia Alonzo, María Gonzales y Jaime Huanca

La revisión de la lingüística aplicada a la enseñanza de lenguas estuvo orientada a establecer cuál podría ser el método o el enfoque más adecuado para nuestro propósito (Breen, 1996 y 1997; Reznicek-Parrado, 2013; Torres, 2011; Cassell, 2016, De León, 2012; Potowski, 2005; Consejo de Europa, 2002; Zanon y Estaire, 2010, Murcia, 1996). Además, se realizó una revisión de los principales métodos de enseñanza del quechua como L2 (Gálvez, 1990; Soto, 1993; Goddenzzi y Vengoa, 1994; Zariquiey y Córdova, 2008). Concluimos que el método que proponemos reunirá características de enseñanza del quechua como L1, así como también algunas estrategias de L2. Por ello lo mejor es establecer varios enfoques pasibles de ser integrados dentro del método, estos fueron el enfoque comunicativo-discursivo, el enfoque por tareas o proyectos, el enfoque de análisis crítico del discurso y el enfoque que nosotros denominamos de contenido contrastivo entre la cultura propia (intracultural) y la cultura foránea (intercultural).

Finalmente, la revisión de los aspectos socioculturales de los pueblos andinos actuales (Degregori, 2000; Pajuelo, 2019; Quijano, 2000; Remy, 2013, entre otros), se realizó para determinar los tópicos más importantes que debían ser considerados como contenidos socioculturales tanto para el ámbito de lo intracultural y lo intercultural.

\subsection{Metodología}

La metodología empleada en este estudio comprendió dos tipos de aproximación. Una primera fue establecer un conjunto de estudios complementarios de tipo exploratorio que correspondían al área disciplinar denominada aplicación lingüística. El objetivo de estos estudios era coadyuvar en determinar las características de los bilingües receptivos.

Para realizar la caracterización del bilingüe receptivo fue necesario ir a la región de Ayacucho, a la ciudad de Huanta a fin de realizar entrevistas al sector de bilingües receptivos elegidos: los estudiantes de los institutos superiores Instituto Superior Tecnológico de Huanta e Instituto Superior Pedagógico «José Salvador Cavero».

El método empleado fue el de casos y el instrumento elaborado fue un cuestionario de preguntas abiertas sobre los temas de caracterización sociolingüística, actitudes lingüísticas, políticas lingüísticas familiares, tipos de bilingüismo, adquisición incompleta de L1 y atrición lingüística.

146 Lengua \& Sociedad. Revista de Lingüística Teórica y Aplicada 
Lineamientos para un método de enseñanza de quechua para bilingües receptivos...

Norma Meneses, Alicia Alonzo, María Gonzales y Jaime Huanca

La segunda aproximación metodológica fue la que se realizó para la revisión de las otras tres áreas disciplinares: lingüística quechua, lingüística aplicada a enseñanza de lenguas y estudios socioculturales de los pueblos andinos.

El método empleado en estas tres áreas fue evaluación crítica de la información bibliográfica en cada área a fin de establecer el estado de la cuestión y sobre esa base determinar los lineamientos teórico-metodológicos del método.

La revisión de los temas sobre la lingüística aplicada a la enseñanza de lenguas estuvo destinada a la selección y fundamentación del método elegido.

Revisar el estado de la cuestión sobre los estudios de lingüística quechua tiene por objetivo determinar los contenidos gramaticales del método, tanto para los aspectos de gramática descriptiva como para los de la gramática normativa.

La revisión de los estudios socioculturales de los pueblos andinos está orientado hacia el establecimiento de los contenidos socioculturales, intraculturales e interculturales del método.

\section{Diagnóstico y caracterización de los usuarios del método}

Situación sociolingüística de estudiantes de nivel superior que son bilingües receptivos de quechua chanka en la ciudad de Huanta, Ayacucho

Los estudiantes bilingües receptivos de quechua de las distintas zonas donde se habla el quechua chanka pertenecen a las regiones de Huancavelica, Ayacucho y Apurímac y serán usuarios de este método de enseñanza-aprendizaje de quechua. Estos estudiantes provienen de realidades lingüísticas relativamente similares pues todos tienen en común ser hijos de hablantes plenos del quechua chanka, la mayoría tienen un entronque directo con las zonas rurales de sus respectivas zonas. Por esta razón, se eligió como zona de estudio a la provincia de Huanta, Ayacucho y como grupo social objetivo a los jóvenes mayores de 18 que estudian en el Instituto Superior Tecnológico de Huanta, el Instituto Superior Pedagógico «José Salvador Cavero» y a la Universidad Nacional Autónoma de Huanta, de reciente creación.

La mayoría de estos alumnos tienen conocimiento de ambas lenguas en algún grado con el castellano como su lengua principal debido al factor educacional. En nuestro país, alcanzar la instrucción terciaria presupone haber superado la secundaria, que solo se brinda en español e iniciar estudios superiores que, de igual manera, se imparte únicamente en esta lengua. En este grupo de alumnos, 
Lineamientos para un método de enseñanza de quechua para bilingües receptivos... Norma Meneses, Alicia Alonzo, María Gonzales y Jaime Huanca

existen distintas formas o tipos de bilingüismo que de manera sucinta podemos mencionar. Existen algunos casos de bilingüismo consecutivo tardío, en los cuales la lengua indígena fue su primera lengua de socialización y aprendieron el castellano más allá de los seis años. También se ha encontrado casos en los cuales el alumno primero adquirió el castellano y luego aprendió el quechua antes de los 10 años debido a situaciones de migración de retorno, tras el término del periodo de violencia política que sufrió la región en las décadas de los '80 y '9o. Sin embargo, se ha podido observar que el grueso de los alumnos presenta un bilingüismo de cuna o nativo por el cual aprendieron ambas lenguas simultáneamente con el quechua como lengua cotidiana de la casa y el castellano como lengua para conversar con el padre y los hermanos. Al ingresar a la escuela primaria el castellano se fue convirtiendo en su lengua más desarrollada y empleada.

En cuanto al castellano, el castellano de los jóvenes presenta los rasgos propios de la variedad adquisicional (interlecto) y regional a los cuales han estado expuesto en la casa y en la ciudad respectivamente. Estas variedades tienen sus peculiaridades en lo fonético, morfosintáctico y léxico ya que estas peculiaridades no son estáticas, sino que se desplazan, penetran o se acomodan en una misma comunidad hablante.

Es importante remarcar que el dominio oral de la lengua originaria en los jóvenes entrevistados se refiere al uso de discursos propios de la dinámica de relación familiar y socioproductiva de las comunidades de donde proceden. Es decir, poseen un conocimiento gramatical y un manejo léxico que les posibilita la comunicación cotidiana básica dentro del hogar y la chacra. Estos jóvenes son conscientes que requieren de mayor desarrollo y práctica de sus competencias orales en los diferentes discursos sociales de mayor complejidad como por ejemplo discutir y proponer alternativas de solución a un problema en quechua. Reconocen que sus competencias son aún más limitadas si se trata de explicar en quechua temas escolares, académicos y de la vida social urbana. Todos señalaron que su paso por la educación secundaria los orientó hacia el empleo del castellano como su lengua principal. También señalaron que percibían que sus familiares y vecinos con menos de 15 años tenían menores competencias lingüísticas que ellos y que incluso los más pequeños solo entendían, mas no hablaban el quechua.

148 Lengua \& Sociedad. Revista de Lingüística Teórica y Aplicada 
Lineamientos para un método de enseñanza de quechua para bilingües receptivos...

Norma Meneses, Alicia Alonzo, María Gonzales y Jaime Huanca

\section{Marco teórico del método de enseñanza de lenguas}

\subsection{La lingüística aplicada a la enseñanza de lenguas}

De manera general, podemos decir que el aprendizaje de lenguas constituye un campo de estudio y de aplicación bastante explorada y trabajada desde mediados del siglo xx. La lingüística aplicada a la enseñanza de lenguas se originó como un quehacer orientado a la enseñanza de lenguas extranjeras en ese periodo. Actualmente, diferencia su actividad en cuatro áreas definidas: 1) la enseñanza de la lengua materna o L1, que se orienta a la enseñanza de la variedad estándar de una lengua en su expresión escrita y oral; 2) la enseñanza de la segunda lengua o L2, que aborda la enseñanza de la lengua y la cultura de un pueblo que comparte el territorio del aprendiz; 3) la enseñanza de la lengua extranjera, extraña o LE, de aquella que no coexiste con la lengua del aprendiz, y 4) la enseñanza de la lengua para propósitos especiales o LPE, que aborda la enseñanza de una competencia específica y no la integridad de las cuatro competencias lingüísticas (escuchar, hablar, leer y escribir).

Existe un conocimiento establecido que todo programa de enseñanza de lenguas debe incluir los siguientes factores:

1. La lengua de enseñanza. métodos son el método clásico o

2. La lengua de instrucción, es decir, se utilizará la lengua de enseñanza también como vehículo instrumental o, existirán apoyos de la lengua del aprendiz.

3. Los niveles de lenguas; cuántos niveles, cuál es el punto de partida del programa y cuál es el punto de llegada.

4. Qué se enseña (gramática, conversación (informal informal) comprensión y producción de textos), nos referimos a los contenidos que se considera debe lograr el aprendiz.

5. Cómo se enseña, es decir, el método que se utilizará.

\subsection{Los métodos de enseñanza de lenguas}

La metodología de enseñanza de lenguas comprende un conjunto de métodos y enfoques de enseñanza-aprendizaje de lenguas como lo señala Breen (1996 y 1997). Los principales métodos son el método clásico o gramatical que se basa en la enseñanza de la gramática de la lengua con abundantes ejercicios de traducción 
Lineamientos para un método de enseñanza de quechua para bilingües receptivos... Norma Meneses, Alicia Alonzo, María Gonzales y Jaime Huanca

y memorización. El método audio-oral, fundamentada en la teoría conductista del psicólogo B. Skinner, se caracteriza por los ejercicios de repetición y memorización de un conjunto de estructuras sintácticas básicas. El método funcional se crea a partir del empleo de la lengua en las distintas funciones sociales del lenguaje (representativa, emotiva, apelativa, fática, estética y metalingüística). Finalmente, el enfoque comunicativo se orienta al desarrollo de competencias comunicativas en situaciones comunicativas cercanas a la realidad. A continuación, desarrollamos este último enfoque, en su versión basada en tareas, pues es el que consideramos la mejor propuesta para el método de fortalecimiento de la L1 de bilingües receptivos.

\subsection{Enfoque comunicativo por tareas}

Es importante señalar que, en la enseñanza de lenguas, desde la aparición del enfoque comunicativo, se diferencia entre el enfoque metodológico y el método de enseñanza de una lengua. De manera general, se puede precisar que el método es la aplicación competente de los supuestos teóricos de una teoría determinada en la resolución de una problema; en tanto que el enfoque es una perspectiva de aproximación que tiene la flexibilidad metodológica de ensayar diversas propuestas teóricas y sus respectivos métodos y técnicas orientándolas a lograr el mismo objetivo de resolver el problema (Breen, 1996 y 1997). En el caso del enfoque comunicativo, su característica es que se orienta al objetivo de lograr que el aprendiz se comunique eficiente y eficazmente empleando la L2 y para ello debe aprender desde el inicio a comunicarse con ella.

Por esta razón, el enfoque comunicativo es una aproximación diferente a la enseñanza de lenguas. Así, más que hablar de métodos, se habla de una forma de concebir la enseñanza donde confluyen distintos métodos y estrategias para la enseñanza de la lengua. A veces es posible que bajo esta etiqueta se empleen estrategias aparentemente opuestas. Por ejemplo, puede haber un distinto tratamiento de la reflexión gramatical en la enseñanza de la lengua: unos la rechazarán totalmente; pero otros la incorporarán de manera tangencial y de apoyo. Todo dependerá si el docente considera que será o no útil para incentivar la comunicación del aprendiz empleando la L2 o LE, según el caso.

Dentro del enfoque comunicativo, se trabajará con lo que se conoce como enseñanza por tareas de comunicación que, para algunos autores, es considerado el intento más adecuado para dar forma concreta al enfoque comunicativo. En

$150 \quad$ Lengua \& Sociedad. Revista de Lingüística Teórica y Aplicada 
Lineamientos para un método de enseñanza de quechua para bilingües receptivos...

Norma Meneses, Alicia Alonzo, María Gonzales y Jaime Huanca

la enseñanza de la segunda lengua Murcia (1996), señala que se debe fomentar procesos «analíticos» de asimilación de la L2. Es decir, la lengua debe ser presentada en el aula como formas naturales y completas de hablar, como discursos que poseen unidad de sentido y de organización retórica, y nunca como oraciones y frases aisladas. La labor de la mente del aprendiz será la de asimilar paulatina e inconscientemente la estructuración semántica, gramatical, textual y discursiva de estas unidades comunicativas. El enfoque comunicativo podrá incorporar si es necesario una estrategia contrastiva para ayudar al aprendiz a la toma de conciencia de las diferencias lingüísticas y/o culturales que se considere importante remarcar. Consideramos que esta misma recomendación resulta absolutamente esencial en la enseñanza de la L1, cuando se enseña la variedad formal, pues se trata de variedades distintas de una lengua.

En esa perspectiva, para la enseñanza de quechua chanka normalizado y modernizado se propone trabajar en un enfoque basado en tareas. Esta es definida según Zanón y Estaire (2010) como una unidad de trabajo que comprende una serie de operaciones cognitivas, de comunicación y socialización, así como el empleo de elementos (lingüísticos y no lingüísticos), para resolver un problema real preestablecido u obtener ciertos resultados mediante el uso de la L2. De esta manera, las «tareas» permitirán dar unidad significativa a las diversas actividades de aprendizaje y comunicación que conllevan, así como tratar como una unidad articulada las diversas destrezas que se despliegan frente a un objetivo de comunicación en una situación comunicativa definida.

\subsubsection{Pasos en el diseño de una unidad de trabajo por «tareas»}

Para la enseñanza de una segunda lengua por tareas, Estaire y Zanon (1990) proponen los siguientes pasos en la planificación de las clases de castellano como segunda lengua:

1. Elección de un tema central que dote de unidad semántica al diseño; generalmente en colaboración con los alumnos y en relación con áreas de su interés: las fiestas de tu ciudad/barrio, la diversión en tu entorno, viajes al extranjero, programas de TV, etc.

2. ${ }^{\circ} \quad$ Especificación de los objetivos de aprendizaje, teniendo presentes el plano conceptual (sistema de la L2), el plano de comunicación (uso de la L2), las estrategias de aprendizaje (de las cuatro macrohabilidades lingüísticas), y los objetivos formativos: por ej. empatía. 
Lineamientos para un método de enseñanza de quechua para bilingües receptivos... Norma Meneses, Alicia Alonzo, María Gonzales y Jaime Huanca

3. ${ }^{\circ}$ Determinación de la/s tarea/s final/es que intencionalmente promoverán la consecución los objetivos propuestos.

$4 .^{\circ} \quad$ Previsión de actividades y pasos posibilitadores para la ejecución de la/s tarea/s final/es. Es decir, actividades de aula o tareas menores para familiarizar a los alumnos con determinados objetivos de aprendizaje, siempre y cuando sean necesarios para la realización de la tarea o tareas finales. Se debe prever incluso el diseño actividades que ayuden a superar las necesidades y carencias específicas y atender las preferencias y los estilos cognitivos de diversos grupos de alumnos.

5. La evaluación. Esta debe contemplar tanto el aprendizaje de los alumnos corno la idoneidad del diseño de instrucción aplicado. La evaluación de los primeros podrá ir asociada al grado de cumplimiento de las tareas finales y al desarrollo de ciertos momentos de los pasos posibilitadores. La evaluación del diseño de instrucción puede ser un enjuiciamiento participativo profesor-alumnos en términos de logros de comunicación.

\subsection{Organización de las competencias lingüísticas por niveles}

Para la determinación de las competencias y niveles de desarrollo del método, se tomó como referencia las competencias propuestas en el Marco Común Europeo de Referencia para las Lenguas (Consejo de Europa, 2002). En este estudio desarrollado para el aprendizaje de lenguas como segunda lengua o como lengua extranjera, se propone que la competencia comunicativa, específicamente relacionada con la lengua, tiene tres componentes: las competencias lingüísticas, las competencias sociolingüísticas y las competencias pragmáticas. Como parte de las competencias lingüísticas, considera la competencia léxica, la gramatical, la semántica, la fonológica, la ortográfica y la ortológica (articulación de pronunciación correcta a partir de la forma escrita).

En este marco, los niveles de dominio de la Estructura de Niveles son 6: A1 (de acceso), A2 (de plataforma), B1 (umbral), B2 (intermedio alto), C1 (dominio operativo eficaz) y $\mathrm{C}_{2}$ (maestría). Nosotros le hemos añadido el nivel $\mathrm{C}_{3}$ que denominamos de sabiduría debido a que el aprendiz es uno que aprende a desarrollar su L1, el quechua, para contribuir a su arraigo en la sociedad como una lengua moderna e instrumento de la construcción de una cultura de la escritura en quechua. Los aprendices son alumnos de institutos superiores que deben contribuir con su escritura académica, administrativa y profesional en quechua, en el futuro.

152 Lengua \& Sociedad. Revista de Lingüística Teórica y Aplicada 
Lineamientos para un método de enseñanza de quechua para bilingües receptivos...

Norma Meneses, Alicia Alonzo, María Gonzales y Jaime Huanca

\begin{tabular}{|c|c|c|}
\hline Nivel & Subnivel & Descripción \\
\hline \multirow[t]{2}{*}{$\begin{array}{l}\text { A } \\
\text { (Usuario } \\
\text { básico) }\end{array}$} & $\begin{array}{l}\text { A1 } \\
\text { (Acceso) }\end{array}$ & $\begin{array}{l}\text { Es capaz de comprender y utilizar expresiones cotidianas } \\
\text { de uso muy frecuente así como frases sencillas destinadas } \\
\text { a satisfacer necesidades de tipo inmediato. Puede } \\
\text { presentarse a sí mismo y a otros, pedir y dar información } \\
\text { personal básica sobre su domicilio, sus pertenencias y } \\
\text { las personas que conoce. Puede relacionarse de forma } \\
\text { elemental siempre que su interlocutor hable despacio y } \\
\text { con claridad y esté dispuesto a cooperar. }\end{array}$ \\
\hline & $\begin{array}{l}\text { A2 } \\
\text { (Plataforma) }\end{array}$ & $\begin{array}{l}\text { Es capaz de comprender frases y expresiones de uso } \\
\text { frecuente relacionadas con áreas de experiencia que } \\
\text { le son especialmente relevantes (información básica } \\
\text { sobre sí mismo y su familia, compras, lugares de interés, } \\
\text { ocupaciones, etc). Sabe comunicarse a la hora de llevar } \\
\text { a cabo tareas simples y cotidianas que no requieran más } \\
\text { que intercambios sencillos y directos de información } \\
\text { sobre cuestiones que le son conocidas o habituales. Sabe } \\
\text { describir en términos sencillos aspectos de su pasado } \\
\text { y su entorno así como cuestiones relacionadas con sus } \\
\text { necesidades inmediatas. }\end{array}$ \\
\hline \multirow[t]{2}{*}{$\begin{array}{l}\text { B } \\
\text { (Usuario } \\
\text { independiente) }\end{array}$} & $\begin{array}{l}\text { B1 } \\
\text { (Intermedio) }\end{array}$ & $\begin{array}{l}\text { Es capaz de comprender los puntos principales de textos } \\
\text { claros y en lengua estándar si tratan sobre cuestiones } \\
\text { que le son conocidas, ya sea en situaciones de trabajo, } \\
\text { de estudio o de ocio. Sabe desenvolverse en la mayor } \\
\text { parte de las situaciones que pueden surgir durante un } \\
\text { viaje por zonas donde se utiliza la lengua. Es capaz de } \\
\text { producir textos sencillos y coherentes sobre temas que } \\
\text { le son familiares o en los que tiene un interés personal. } \\
\text { Puede describir experiencias, acontecimientos, deseos y } \\
\text { aspiraciones, así como justificar brevemente sus opiniones } \\
\text { o explicar sus planes. }\end{array}$ \\
\hline & $\begin{array}{l}\text { B2 } \\
\text { (Intermedio } \\
\text { alto) }\end{array}$ & $\begin{array}{l}\text { Es capaz de entender las ideas principales de textos } \\
\text { complejos que traten de temas tanto concretos como } \\
\text { abstractos, incluso si son de carácter técnico siempre } \\
\text { que estén dentro de su campo de especialización. Puede } \\
\text { relacionarse con hablantes nativos con un grado suficiente } \\
\text { de fluidez y naturalidad de modo que la comunicación } \\
\text { se realice sin esfuerzo por parte de ninguno de los } \\
\text { interlocutores. Puede producir textos claros y detallados } \\
\text { sobre temas diversos así como defender un punto de vista } \\
\text { sobre temas generales indicando los pros y los contras de } \\
\text { las distintas opciones. }\end{array}$ \\
\hline
\end{tabular}


Lineamientos para un método de enseñanza de quechua para bilingües receptivos... Norma Meneses, Alicia Alonzo, María Gonzales y Jaime Huanca

\begin{tabular}{|c|c|c|}
\hline Nivel & Subnivel & Descripción \\
\hline \multirow[t]{3}{*}{$\begin{array}{l}\text { C } \\
\text { (Usuario } \\
\text { competente) }\end{array}$} & $\begin{array}{l}\text { C1 } \\
\text { (Dominio } \\
\text { operativo } \\
\text { eficaz) }\end{array}$ & $\begin{array}{l}\text { Es capaz de comprender una amplia variedad de textos } \\
\text { extensos y con cierto nivel de exigencia, así como } \\
\text { reconocer en ellos sentidos implícitos. Sabe expresarse } \\
\text { de forma fluida y espontánea sin muestras muy evidentes } \\
\text { de esfuerzo para encontrar la expresión adecuada. Puede } \\
\text { hacer un uso flexible y efectivo del idioma para fines } \\
\text { sociales, académicos y profesionales. Puede producir } \\
\text { textos claros, bien estructurados y detallados sobre temas } \\
\text { de cierta complejidad, mostrando un uso correcto de los } \\
\text { mecanismos de organización, articulación y cohesión } \\
\text { del texto. }\end{array}$ \\
\hline & $\begin{array}{l}\text { C2 } \\
\text { (Maestría) }\end{array}$ & $\begin{array}{l}\text { Es capaz de comprender con facilidad prácticamente } \\
\text { todo lo que oye o lee. Sabe reconstruir la información } \\
\text { y los argumentos procedentes de diversas fuentes, } \\
\text { ya sean en lengua hablada o escrita, y presentarlos } \\
\text { de manera coherente y resumida. Puede expresarse } \\
\text { espontáneamente, con gran fluidez y con un grado de } \\
\text { precisión que le permite diferenciar pequeños matices de } \\
\text { significado incluso en situaciones de mayor complejidad. }\end{array}$ \\
\hline & $\begin{array}{l}\text { C3 } \\
\text { (Sabiduría) }\end{array}$ & $\begin{array}{l}\text { Es consciente del contenido intracultural e intercultural } \\
\text { de los textos que lee y redacta. Es consciente de su } \\
\text { contribución a la creación de una tradición escrita del } \\
\text { quechua en todo tipo de género discursivos y textuales. }\end{array}$ \\
\hline
\end{tabular}

\section{Nuestra propuesta de lineamientos para el método de enseñanza de quechua como L1 para bilingües receptivos en quechua}

Se explicita que el método que se pretende diseñar va dirigido a bilingües receptivos en quechua que tienen una adquisición incompleta de una de sus lenguas maternas, el quechua, cuya funcionalidad activa no pudieron completar por diversos motivos habiéndose quedado en distintos niveles de adquisición: inicial, intermedio y avanzado. La característica de este tipo de bilingües es que sus competencias comprensivas superan a sus competencias productivas. Es decir, comprenden lo que se les dice en quechua pero no se sienten capaces de responder bien en esta lengua.

154 Lengua \& Sociedad. Revista de Lingüística Teórica y Aplicada 
Lineamientos para un método de enseñanza de quechua para bilingües receptivos...

Norma Meneses, Alicia Alonzo, María Gonzales y Jaime Huanca

\subsection{Niveles de dominio de lengua}

Trabajaremos con nueve ciclos de enseñanza lengua agrupados en tres niveles, que van desde el básico I hasta el avanzado III. Es importante recalcar que estos niveles no hacen referencia a principiantes/avanzados en el aprendizaje de una 2da lengua o de una lengua extranjera ya que estos parten de un conocimiento cero de la lengua meta.

Nuestro punto de partida son hablantes bilingües quechua-castellano, con habilidades comunicativas en la lengua quechua, que necesitan profundizar su conocimiento en la misma, para desenvolverse en situaciones que van más allá de lo cotidiano; es decir, en un lenguaje más formal en el nivel oral y también, deben aprender a redactar distintos tipos de documentos.

Los contenidos que se enseñan son aspectos comunicativos y gramaticales inmersos en la conversación informal, formal, y en la comprensión y producción de textos.

\subsection{Competencias meta}

El método se orienta al afianzamiento de las capacidades comunicativas de los aprendices tanto de comprensión (escuchar, leer) como de producción (hablar y escribir) en la lengua quechua. Además, se tomará en cuenta el desarrollo de las siguientes competencias que consideramos transversales en el currículo de este programa de enseñanza:

1. La comunicación asertiva y cordial

2. La colaboración

3. El pensamiento crítico

4. La creatividad

5. La alfabetización informacional

6. La alfabetización digital

7. El compromiso étnico o intracultural

8. El compromiso intercultural.

La comunicación debe conllevar la incorporación de los usos propios de la lengua que conduzcan a una conversación no solo eficaz, sino también asertiva y cordial. La colaboración es una competencia orientada a rescatar los valores de solidaridad y reciprocidad en el trabajo colectivo y en equipos de pares, propios de la cultura andina. El pensamiento crítico debe conducir a la reflexión y valoración de las condiciones y factores presentes en una situación comunicativa 
Lineamientos para un método de enseñanza de quechua para bilingües receptivos... Norma Meneses, Alicia Alonzo, María Gonzales y Jaime Huanca

y de los propósitos declarados y ulteriores de los mensajes. La creatividad es una capacidad que se orientará al desarrollo de un pensamiento innovador que sepa valorar y extraer nuevas ideas a partir de la diversidad y la variedad de los elementos de la realidad. La alfabetización informacional debe capacitar al aprendiz a saber cuándo y por qué necesita la información, dónde encontrarla, y cómo evaluarla, utilizarla y comunicarla de manera ética. Esta capacidad es considerada por la OCDE un prerrequisito para acceder a la Sociedad de la Información y del Conocimiento y es reconocida como parte de los derechos básicos de la Humanidad para un asegurar un aprendizaje permanente.

Directamente ligada a estas competencias se desarrolla la competencia digital ya que la construcción de una cultura de la escritura en quechua deberá adaptarse a las formas y medios de la comunicación actual que se fundamenta en el empleo de las TIC y las redes sociales digitales. La competencia digital es definida el 2005 por la Unión Europea como sigue:

Entraña el uso seguro y crítico de las tecnologías de la sociedad de la información (TSI) para el trabajo, el ocio y la comunicación. Se sustenta en las competencias básicas en materia TsI: el uso de ordenadores para obtener, evaluar, almacenar, producir, presentar e intercambiar información, y comunicarse y participar en redes de colaboración a través de Internet. (Unión Europea, 2005, p.18)

Finalmente, proponemos dos competencias: el compromiso étnico o intracultural y el compromiso intercultural. El primero lo definimos como la motivación y voluntad de contribuir con el afianzamiento y desarrollo de su colectivo cultural. En el caso del método propuesto, el aprendiz deberá estar muy motivado a contribuir con el arraigo de un quechua ayacuchano estandarizado y modernizado, así como con la construcción de una tradición escrita en lengua quechua. No solo debe orientarse a una autoidentificación étnica pasiva, sino a una más activa y de liderazgo cultural. El compromiso intercultural lo proponemos como una capacidad del aprendiz orientada a aproximarse a las otras culturas de su entorno con genuino afán de conocimiento, valoración y empatía.

156 Lengua \& Sociedad. Revista de Lingüística Teórica y Aplicada 
Lineamientos para un método de enseñanza de quechua para bilingües receptivos... Norma Meneses, Alicia Alonzo, María Gonzales y Jaime Huanca

\subsection{Géneros discursivos}

Se tomó de base la teoría del análisis del discurso expuesta por Bajtin (1989) y Alexopoulou (2011) y las propuestas conceptuales sobre la escritura de Cassany (2016), aunada a la información de los estudios culturales andinos más nuestras propias reflexiones, para establecer los géneros discursivos que se desarrollarán:

1. Discurso de la vida cotidiana familiar rural y urbana

2. Discurso de la vida comunal y de barrio urbano

3. Discurso de la actividad agropastoril de valle interandino en temporada de secas y de lluvias.

4. Discurso de la actividad agropastoril de zona altoandina en temporada de secas y de lluvias.

5. Discurso de otras actividades productivas: artesanías

6. Discurso de la actividad social de la ciudad

7. Discurso de la actividad comercial tradicional

8. Discurso de la actividad socioeconómica urbana

9. Discurso de las creencias, principios cognoscitivos y valores ancestrales

10. Discurso de las creencias, principios y valores de la cultura occidental cristiana

11. Discurso de la salud y la etnomedicina

12. Discurso de la sabiduría y la tecnología ancestrales

13. Discurso de la asociación, la organización social y el derecho ancestrales

14. Discurso de la asociación, organización sociopolítica y el derecho en la sociedad oficial

15. Discurso de la actividad de entretenimiento en la comunidad

16. Discurso del entretenimiento en la sociedad oficial

17. Discurso de la actividad académica

18. Discurso de la ciencia y la tecnología modernas

19. Discurso de la aproximación de variantes de la cultura andina y de sus lenguas: quechua norteño, quechua central, quechua cusqueño, quechua ecuatoriano, quechua boliviano, argentino.

20. Discurso de aproximación a la cultura andina Aru: el aimara y el jaqaru

21. Discurso de la exploración de las culturas vecinas en la región de Ayacucho: ashaninka y criolla

22. Discurso de la exploración de pueblos y culturas lejanas: Europa, EEUU, China, Japón, India, etc... 
Lineamientos para un método de enseñanza de quechua para bilingües receptivos... Norma Meneses, Alicia Alonzo, María Gonzales y Jaime Huanca

\subsection{Situaciones comunicativas y lecciones}

Las situaciones comunicativas estarán enmarcadas dentro de alguno de los discursos mencionados.

Se tendrá 3 niveles: inicial, intermedio y avanzado. Dentro de cada nivel habrá 3 subniveles. Cada subnivel presentará cuatro lecciones. Cada lección estará formada por los siguientes rubros:

1. Presentación del tema (Situación comunicativa: un diálogo modelo)

2. Ejercicios de oralidad, basados en el diálogo modelo y la situación comunicativa

3. Lectura de textos. Presentación de un texto. Preguntas y ejercicios de comprensión que incluye el reconocimiento del léxico.

4. Retroalimentación de aspectos gramaticales en el uso de la lengua. Se desarrollará junto con la comprensión lectora. A partir del análisis del género textual y tipo de texto.

5. Producción escrita. Los géneros discursivos y textuales a desarrollar deben estar relacionados con el texto presentado para la comprensión de lectura.

6. Ejercicio final de refuerzo. Debe promover el ejercicio de las capacidades comunicativas aprendidas.

7. Test (Evaluación) para pasar a siguiente lección.

8. Glosario. Cada lección deberá presentar el listado de palabras estandarizadas y de neologismos empleados en la lección. Estas entradas deberán ser explicados para no desconcertar al estudiante, pues hay que recordar que entienden el significado original de esas nuevas palabras.

\subsection{Algunos contenidos sobre el uso de la lengua:}

\subsubsection{Contenidos gramaticales:}

- El discurso: modos y géneros discursivos

- El texto, macroestructura y microestructura, propiedades y clases

- El paratexto y el intertexto

- La oración: unidad mínima de la comunicación

- Clases de oraciones por la intención comunicativa:

- La expresión de la certidumbre informativa

158 Lengua \& Sociedad. Revista de Lingüística Teórica y Aplicada 
Lineamientos para un método de enseñanza de quechua para bilingües receptivos... Norma Meneses, Alicia Alonzo, María Gonzales y Jaime Huanca

- La expresión de la inseguridad informativa

- La expresión del deseo o ganas de algo o de hacer algo

- La expresión de la ignorancia

- La expresión de la exhortación

- La expresión de la emoción

- La expresión de la calidez o cordialidad

- El uso de los marcadores independientes o enclíticos

- La oración simple. Límites. El uso del punto y la mayúscula. Inicio de oración

- Orden de los Constituyentes (complementos)

- La frase nominal sujeto. Concordancia. Orden (determinante-núcleo)

- La formación de sustantivos y adjetivos por derivación

- La frase preposicional de la frase nominal (sujeto)

- La frase verbal. Uso de los verbos

- La formación de verbos y adverbios por derivación

- La FN de la FV (objeto directo e indirecto o primario y secundario)

- El uso de los sufijos transicionales de persona actora y de objeto

- Los circunstanciales: de locación, de finalidad, de compañía, de instrumento...)

- Oraciones compuestas subordinadas

- Oraciones compuestas coordinadas

- Las palabras: clases

- Formación de palabras

\subsubsection{Contenidos de Estandarización Linguiística}

Están orientados hacia la estandarización de un quechua sureño.

- Estandarización fonológica con el quechua de Cusco, excepto glotalizadas y aspiradas

- Estandarización morfológica con el quechua cusqueño en base a las formas del protoquechua

- Estandarización léxica con el quechua de Cusco 
Lineamientos para un método de enseñanza de quechua para bilingües receptivos... Norma Meneses, Alicia Alonzo, María Gonzales y Jaime Huanca

\subsubsection{Contenidos de corrección idiomática del quechua}

- Alfabeto: normas de ortografía

- Reglas de uso de las mayúsculas

- Reglas de separación silábica

- Reglas de uso de la tilde

- Reglas de uso de los signos de puntuación (punto, coma, punto y coma, dos puntos, puntos suspensivos, comillas, paréntesis, raya, guion)

\subsubsection{Contenidos de modernización lingüística}

- Neologismos: proceso de formación de palabras nuevas:

- Procesos morfológicos

- Procesos semánticos

- Colocaciones nominales, verbales y adverbiales

- Lexías o frases hechas

- Préstamos arraigados

\section{Conclusiones}

Ha sido posible establecer un conjunto de lineamientos teóricos y metodológicos que permitirán la elaboración de un método de enseñanza-aprendizaje de quechua para bilingues receptivos en quechua. Estos lineamientos se encuentran fundamentados en los estudios de la lingüística quechua, de aplicación lingüística y de lingüística aplicada a la enseñanza de lenguas, así como, en los estudios filosóficos, sociales, antropológicos y económicos sobre los pueblos andinos.

\subsection{Luego de evaluar el conjunto de estudios de lingüística quechua, podemos concluir lo siguiente}

Los estudios de descripción gramatical del quechua son bastante numerosos, especialmente en los aspectos de la fonología, morfología y sintaxis de la frase y la oración simple. No obstante, se necesita completar las investigaciones sobre la sintaxis de la oración simple y emprender el estudio sobre la oración compuesta para dilucidar las formas de expresión de la coordinación y la subordinación. Así mismo, se necesita contar con los estudios sobre semántica y lexicología del

$160 \quad$ Lengua \& Sociedad. Revista de Lingüística Teórica y Aplicada 
Lineamientos para un método de enseñanza de quechua para bilingües receptivos...

Norma Meneses, Alicia Alonzo, María Gonzales y Jaime Huanca

quechua. Hay una necesidad urgente de emprender la modernización lingüística del quechua tanto en los aspectos léxicos como en los temas discursivo-textuales y pragmáticos a fin de asegurar el desarrollo del quechua como una lengua moderna.

Hay necesidad de realizar más estudios sobre el léxico quechua para identificar el universo vocabular de cada área o actividad de la cultura andina, a fin de que sirvan de base para los procedimientos de modernización lingüística de esta lengua.

Se advierte que la planificación lingüística del quechua está aún incompleta tanto en la planificación del estatus y del corpus de la lengua quechua. Se necesita dar cuenta de los procedimientos de la planificación del corpus de esta lengua. Hasta el momento solo se ha avanzado en la determinación de los alfabetos para la escritura de los seis dialectos más importantes del quechua. Aún falta realizar la estandarización lingüística de los aspectos morfológicos y sintácticos dentro de estas grandes áreas dialectales. En cuanto a la corrección idiomática de la escritura, falta establecer las reglas de uso de mayúsculas, uso de la tilde, uso de los signos de puntuación, separación silábica, precisión léxica, estructuración textual.

Otro aspecto pendiente y muy urgente de la planificación del corpus del quechua es la modernización léxica. Aún no se cuentan con léxicos modernizados del quechua que den cuenta de todas las actividades de la sociedad moderna actual. Existen propuestas individuales realizadas por autores de textos de enseñanza del quechua, así como de autores de textos escolares para la educación intercultural bilingüe (ЕІB), pero estos esfuerzos no están coordinados ni articulados entre sí y por ello estos textos son de poco uso y aceptación por no ser entendibles para los alumnos y sus padres e incluso para los propios docentes de aula. Esto se hace evidente en las escuelas EIB a cargo del Ministerio de Educación y de las oNG.

Lo más urgente para la elaboración del método es contar con un léxico modernizado de términos gramaticales y con un léxico de la actividad pedagógica de enseñanza de lenguas, ambos escritos en quechua.

\subsection{De los estudios complementarios realizados dentro del área de aplicación lingüística, se desprende lo siguiente}

Los bilingues quechua-español receptivos en quechua conforman un grupo numeroso y creciente en el Perú. Los jóvenes entrevistados, alumnos de los institutos superiores Instituto Superior Tecnológico de Huanta e Instituto Superior Pedagógico José Salvador Cavero, presentan una buena comprensión oral (escuchar), en 
Lineamientos para un método de enseñanza de quechua para bilingües receptivos... Norma Meneses, Alicia Alonzo, María Gonzales y Jaime Huanca

contraste con niveles más bajos de producción lingüística en quechua (hablar), por ello, difieren de los aprendices de quechua como segunda lengua y lengua extranjera porque estos acceden al programa de enseñanza-aprendizaje del quechua sin ningún conocimiento de esta lengua. Por lo tanto, el problema de la atrición lingüística, o desgaste de la capacidad de hablar la lengua materna por falta de uso, no es un problema significativo ya que la ciudad de Huanta aún es una zona de pleno bilingüismo social.

Se ha podido determinar que el problema más notorio en este grupo de alumnos es el de la adquisición incompleta de L1 ya que el quechua solo es empleado en el ámbito familiar. Al ingresar a sus estudios de educación básica (inicial, primaria y secundaria), fueron expuestos únicamente al castellano. En casa se les apoyó en el manejo de esta lengua en desmedro del uso del quechua. Por esta razón, no continuaron con el refinamiento de su L1 a mayores niveles de usos discursivos y textuales de mayor complejidad, incluso dentro de la temática cotidiana familiar.

La mayoría de estos estudiantes presentan tanto bilinguismo consecutivo temprano (adquisición de L2 castellano entre 3 a 10 años) como bilingüismo de cuna (adquisicion de dos lenguas maternas simultáneamente). Es decir, que la exposición al castellano ocurre en la temprana infancia. Esto difiere del bilinguismo de sus padres que usualmente es un bilingüismo consecutivo tardío ya que su exposición al castellano fue a los 6 o 7 años con el ingreso a la primaria, pues antes no era obligatorio ni había muchas centros de educación inicial.

Estos estudiantes provienen de hogares de origen rural que luego migraron a la ciudad de Huanta durante las dos decadas de violencia política de los 80 y 9o, que afectó duramente a la region de Ayacucho. Se evidencia que sus padres tomaron decisiones acerca del tratamiento de las lenguas quechua y castellano en el seno del hogar. Estas decisiones conforman una política lingüística familiar que se expresa en a) su negativa a matricular a sus hijos en escuelas de Educación Intercultural Bilingüe (EIB), b) distribuir los roles de portadores de la lengua castellana (padres y hermanos mayores) y de lengua quechua (abuelos) en el seno del hogar, c) desincentivar el uso del quechua por los niños dentro de la casa, permitiendo la comunicación diaural con los abuelos que son bilingües pasivos en español, d) exigir el uso del castellano cuando la persona le habla en castellano. 
Lineamientos para un método de enseñanza de quechua para bilingües receptivos...

Norma Meneses, Alicia Alonzo, María Gonzales y Jaime Huanca

5.3 De la evaluación de los estudios de lingüística aplicada a la enseñanza de lenguas, se concluye lo siguiente:

El método que se diseña es un método de enseñanza de quechua como lengua materna o L1 para estudiantes que son bilingües receptivos en quechua, con el objetivo de convertirse en bilingües activos quechua-español. Es decir, se orienta a la recuperación del dominio avanzado del quechua. Si bien el método tiene un carácter básico de enseñanza de L1, se añadirán algunas estrategias propias de la enseñanza de L2 debido a que los destinarios van a tener como lengua meta una variedad estandarizada, modernizada y normalizada de quechua ayacuchano.

El método elegido es más bien un enfoque pedagógico: comunicativo-discursivo centrado en tareas. Cada tarea esta relacionada a una situacion comunicativa y tiene objetivos precisos de comunicación en torno a los cuales se desarrollan las habilidades y la informacion gramatical.

Se empleará un enfoque discursivo crítico, a fin de que el aprendiz desarrolle su capacidad de reflexión para identificar las intenciones comunicativas y los mensajes culturales e ideológicos que conllevan los textos.

Se aplicará también un enfoque discursivo contrastivo presentando en los contenidos la información intracultural e intercultural de las actividades humanas. Es decir, en el contenido de las lecciones afianzará en el alumno el conocimiento de su propia cultura (intracultural) y se lo aproximará al conocimiento de la cultura occidental moderna (intercultural) incidiendo en las semejanzas y en las diferencias culturales. Se abordará aspectos de la cultura material y espiritual de ambas sociedades.

\subsection{De la revisión de los aspectos socioculturales propios de los pueblos andinos actuales que deben ser considerados en los contenidos socioculturales del método a elaborar se ha considerado lo siguiente:}

Poner acento en la información intracultural de los pueblos andinos referido al conocimiento de a) su territorio comunal y los pisos altitudinales bajo su control, b) la información de la actividad económica y social basada en la diferencia entre comunidades de valle interandino y comunidades de zona altoandina, c) la información económica y social distribuida según las dos grandes estaciones climáticas del pais: la temporada de secas de mayo a noviembre y la temporada de lluvias de diciembre a abril, d) la información socioeconómica basada en la diferencia entre 
Lineamientos para un método de enseñanza de quechua para bilingües receptivos... Norma Meneses, Alicia Alonzo, María Gonzales y Jaime Huanca

lo rural y lo urbano de la region y e) la información sociocultural y económica de interacción entre población residente en el territorio ancestral y la población migrante a otras regiones del pais y a otras naciones del mundo.

Desarrollar los retos de la modernización de los pueblos andinos. Mostrar que la cultura andina ha permitido a los migrantes andinos posicionarse exitosamente en la sociedad oficial, mediante actividades de emprendimiento económico exitoso. Desarrollar los principios cognoscitivos y los valores culturales que constituyen el núcleo de la cultura andina. Mostrar la apropiación de los elementos económicos y tecnológicos de la modernidad que hacen los pueblos andinos.

Presentar la unidad cultural andina de los pueblos quechuas de los países vecinos de Colombia, Ecuador, Bolivia, Argentina.

Aproximar al conocimiento de las culturas en contacto cotidiano con la cultura de los pueblos andinos: las culturas amazónicas y las culturas criollas o mestizas del país.

Aproximar al conocimiento de las culturas lejanas del Mundo. Se empezará con las grandes culturas matrices del planeta que tienen más de 5000 años de continuidad cultural dentro de las cuales se ubica la cultura andina: Sumeria, Egipto, India, China y Maya.

Dar a conocer los aportes de la cultura occidental de Europa y Estados Unidos.

\section{Recomendaciones}

Por las conclusiones anteriores, es necesario especificar los aspectos que necesitan ser abordados en futuras investigaciones:

- Incrementar los estudios de lingüística que aborden otros aspectos que cuentan con poca o ninguna información en el conocimiento de la lengua quechua: sintaxis, semántica, lexicología, discurso y texto, pragmática.

- Realizar investigaciones que den cuenta de las necesidades de información para una adecuada lingüística aplicada a la lengua quechua. Se necesita desarrollar todo tipo de léxicos referidos a las actividades socioeconómicas y culturales de los pueblos andinos.

- Es urgente contar con estudios con participación de colectivos de intelectuales andinos para realizar propuestas de modernización del léxico quechua, a fin de contribuir con la tarea incompleta de la planificación de corpus de la lengua quechua.

164 Lengua \& Sociedad. Revista de Lingüística Teórica y Aplicada 
Lineamientos para un método de enseñanza de quechua para bilingües receptivos...

Norma Meneses, Alicia Alonzo, María Gonzales y Jaime Huanca

- Se necesita elaborar para el quechua un léxico de términos gramaticales y un léxico de términos de la enseñanza de lenguas. Estos documentos son esenciales para asegurar el éxito de todo texto de enseñanza del quechua tanto como lengua materna, segunda lengua y lengua extranjera.

- Se necesita elaborar un diccionario del quechua sureño moderno, donde se reúnan no solo los términos tradicionales estandarizados del quechua de Ayacucho y Cusco, sino también los neologismos creados.

\section{Referencias Bibliográficas}

Alexopoulou, A. (2011). El enfoque basado en los géneros textuales y la evaluación de la competencia discursiva. En Javier de Santiago-Guervós, Hanne Bongaerts, Jorge Juan Sánchez Iglesias y Marta Seseña Gómez (Coords.), Del texto a la lengua: La aplicación de los textos a la enseñanza-aprendizaje del español L2-LE (Vol 1; pp. 97-110). España: Asociación para la Enseñanza del Español como Lengua Extranjera. Disponible en https://dialnet.unirioja.es/ servlet/articulo? codigo $=5419275$

Bajtin, M. (1989). El problema de los géneros discursivos. México: Siglo xxI.

Breen, M. (1997). Paradigmas contemporáneos en el diseño de programas de enseñanza de lenguas (II). Signos. Teoría y Práctica de la Educación, 20, 52-73. Disponible en http://www.quadernsdigitals.net/index.php?accionMenu=hemeroteca.VisualizaArticuloıU.visualiza\&articulo_id $=687$

Breen, M. (1996). Paradigmas contemporáneos en el diseño de programas de enseñanza de lenguas (I). Signos. Teoría y Práctica de la Educación, 19, 42-49. Disponible en http://www.quadernsdigitals.net/index.php?accionMenu=hemeroteca.VisualizaArticuloIU.visualiza\&articulo_id=680

Calvo, J. (1993). Pragmática y gramática del Quechua cuzqueño. Cuzco: Centro de Estudios Andinos Bartolomé de las Casas.

Cassany, D. (2016). Los retos de enseñar a escribir en el s. XXI [Diapositivas]. Disponible en https://www.upf.edu/documents/2853238/2856910/Meridao6. pdf/da6f9667-3d93-47d8-82d2-498a027of6bf

Cassell, H. (2016). Hablantes de Herencia (H. H.) de español en las clases de idiomas extranjeros en la escuela secundaria: Los maestros y su preparación (Tesis de maestría en Educación y Desarrollo Humano), The College At Brockport State 
Lineamientos para un método de enseñanza de quechua para bilingües receptivos...

Norma Meneses, Alicia Alonzo, María Gonzales y Jaime Huanca

University of New York, Estados Unidos. Disponible en http://digitalcommons. brockport.edu/cgi/viewcontent.cgi?article $=1701 \&$ context $=$ ehd_theses

Cerron-Palomino, R. (1987). Lingüistica quechua. Cusco: Centro de Estudios Regionales Andinos Bartolomé de las Casas.

Consejo de Europa. (2002). Marco Común Europeo de Referencia para Lenguas: Aprendizaje, enseñanza y evaluación. España: Secretaria General Técnica del Ministerio de Educación, Cultura y Deporte - Subdirección General de Información y publicaciones, y Grupo Anaya. Disponible en https://cvc. cervantes.es/ensenanza/biblioteca_ele/marco/cvc_mer.pdf

Cusihuamán, A. (1976). Gramática del quechua. Cusco-Collao. Lima: Instituto de Estudios Peruanos - Ministerio de Educación.

Davies, A. (2007). An Introduction to Applied Linguistics. Edinburgh: Edinburgh University. Disponible en https://pasca.uns.ac.id/s3linguistik/wp-content/ uploads/sites/44/2016/10/an-introduction-to-applied-linguistics.pdf

Degregori, C. (Ed.). (2000). No hay país más diverso. Compendio de antropología Peruana. Lima: Instituto de Estudios Peruanos - Red para el Desarrollo de las Ciencias Sociales en el Perú.

Gálvez, I. (1990). Quechua Ayacuchano Curso I. Lima: Extramuros.

Gleich, U. von (2014). Nueva dinámica en el bilingüismo ayacuchano. Indiana, 33(1), 133-159. https://doi.org/10.18441/ind.v33i1.133-159

Goddenzzi, J. y Vengoa. J. (1994). Runasimimanta yuyaychakusun: manual de lingüística quechua para bilingües. Cusco.: Asociación Pukllasunchis - Centro de Estudios Regionales Andinos Bartolomé de las Casas.

Heijas, T. (2014). ¿Ser y hablar quechua? La realidad sociolingüística de Ayacucho desde la visión subjetiva de los jóvenes indígenas: Ideologías e identidades en el discurso metalingüístico (Trabajo de fin de máster en Filología Española). Universidad de Helsinki, Finlandia. Disponible en http://urn.fi/ URN:NBN:fi-fe201502121593

León, V. de. (2012). El español de los hablantes de la lengua de herencia de los Estados Unidos: una investigación bibliográfica de las características lexicales y morfosintácticas (Tesis de bachiller). Universidad de Utrecht, Países Bajos. Disponible en https://dspace.library.uu.nl/handle/1874/254243

Murcia, M. (1996). Tareas de comunicación y planificación de la enseñanza de idiomas. Signos Teoría y Práctica de la Educación, 1. Disponible en http://www. quadernsdigitals.net/datos_web/hemeroteca/r_3/nr_32/a_544/544.html 
Lineamientos para un método de enseñanza de quechua para bilingües receptivos...

Norma Meneses, Alicia Alonzo, María Gonzales y Jaime Huanca

Pajuelo, R. (2019). Trayectorias comunales: Cambios y continuidades en comunidades campesinas e indígenas del Sur Andino. Lima: Grupo Propuesta Ciudadana. Disponible en https://propuestaciudadana.org.pe/wp-content/ uploads/2019/10/Trayectorias-comunales.-Cambios-y-continuidades-encomunidades-campesinas-e-ind\%C3\%ADgenas-del-Sur-Andino.pdf

Parker, G. (1965). Gramática del quechua de Ayacucho. Lima: Universidad Nacional Mayor de San Marcos.

Potowski, K. (2005). Fundamentos para la enseñanza del español para hispanohablantes en Estados Unidos. Madrid: Arco Libros.

Quijano, A. (2000). Colonialidad del poder, eurocentrismo y América Latina. En Edgardo Lander (Comp.), La colonialidad del saber: eurocentrismo y ciencias sociales. Perspectivas Latinoamericanas. Argentina: Consejo Latinoamericano de Ciencias Sociales (Clacso). Disponible en http://bibliotecavirtual.clacso.org. ar/ar/libros/lander/quijano.rtf

Remy, M. (2013). Historia de las comunidades indígenas y campesinas del Perú. Lima: Instituto de Estudios Peruanos.

Reznicek-Parrado, L. (2013). Pedagogía de Hablantes de Herencia: implicaciones para el entrenamiento de instructores al nivel universitario (Tesis de maestría). Universidad de Nebraska, Estados Unidos. Disponible en http://digitalcommons.unl.edu/cgi/viewcontent.cgi? article $=1016 \&$ context $=$ modlangdiss

Seton, B. y Schmid, M. (2016). Multi-competence and First Language Attrition. Disponible en http://repository.essex.ac.uk/16768/1/seton\%20\%26\%20schmid. pdf

Sicra, I. (2006). Enseñanza de lengua indígena e interculturalidad: ¿entre la realidad y el deseo? Investigación sobre la enseñanza del quechua en dos colegios particulares de Cochabamba. Cochabamba: Programa de Formació en Educación Intercultural Bilingüe para los Países Bajos Andinos proeıв Andes. Disponible en http://bvirtual.proeibandes.org/bvirtual/docs/ens_lengua_indigena.pdf

Solís, G. y Chacón, J. (1989). Lingüística y gramática runasimi Chanka. Lima: UNESCO-AGFUND.

Soto, C. (1993). Manual de Quechua. Lima: Instituto de Estudios Peruanos.

Soto, C. (1976). Gramática del quechua Ayacucho-Chanka. Lima.: Instituto de Estudios Peruanos - Ministerio de Educación.

Suárez, J. (2002). Un caso de multiculturalidad y bilingüismo: la enseñanza del español a hijos de migrantes hispanos en Estados Unidos. Actas del XIII Congreso Internacional de la Asociación para la Enseñanza del Español como Lengua 
Lineamientos para un método de enseñanza de quechua para bilingües receptivos...

Norma Meneses, Alicia Alonzo, María Gonzales y Jaime Huanca

Extranjera (pp. 817-824). Disponible en http://cvc.cervantes.es/ensenanza/ biblioteca_ele/asele/pdf/13/13_0817.pdf

Szupica-Pyrzanowska, M. (2016). Language Attrition: Implications for Second/

Foreign Language Acquisition. Lingwistyka Stosowana, 16(1), 109-120. Disponible en http://www.ls.uw.edu.pl/documents/7276721/13368455/10+lingwistyka+stosowana+16+malgorzta+szupica-pyrzanowska.pdf

Torres, A. (2011). La enseñanza del español a los hispanohablantes de herencia de los Estados Unidos. Normas. Revista de Estudios Lingüísticos Hispánicos, 1, 133-150. Disponible en http://roderic.uv.es/bitstream/handle/10550/24982/133-150. pdf? sequence $=1$ \&isallowed $=y$

Zanon, J. y Estaire, S. (2010). El diseño de unidades didácticas mediante tareas: principios y desarrollo. MarcoEle, 11, 410-418. Disponible en https://marcoele. com/descargas/navas/21.zanon-estaire.pdf

Zariquiey, R. y Córdova, G. (2008). Qayna, kunan, paqarin. Una introducción práctica al quechua chanca. En: http://repositorio.pucp.edu.pe/index/ handle/123456789/134454 